\title{
CD1, MR1, NKT, and MAIT: evolution and origins of non-peptidic antigen recognition by $T$ lymphocytes
}

\author{
Dirk M. Zajonc ${ }^{1,2}$ - Martin F. Flajnik ${ }^{3}$
}

Received: 12 July 2016 / Accepted: 12 July 2016 /Published online: 1 August 2016

(C) Springer-Verlag Berlin Heidelberg 2016

T cell-mediated immunity against infection and tumors is a central feature of the adaptive immune system. Identifying antigens that induce $\mathrm{T}$ cell activation, and understanding the mechanism of antigen recognition and subsequent activation, are crucial for developing therapeutic strategies aimed at modulating T cell responses. While peptides presented by the polymorphic major histocompatibility complex (MHC) class I and II antigen-presenting molecules provide the majority of antigens recognized by alpha/beta TCR-bearing T cells, other chemical classes of antigens have been identified in the past 20 years that are presented mainly to "innate T cells." These antigens include lipids and glycolipids presented by the CD1 family, and, more recently, microbial vitamin B metabolites presented by MR1. T cells restricted by these class I molecules often show innate-like and unique characteristics, such as either having a conserved TCR rearrangement (semi-invariant type I NKT cells and MAIT cells), a restricted T cell repertoire (CD1b-restricted GEM T cells), or a mode of T cell activation that is independent of direct antigen contacts (CD1aautoreactive $\mathrm{T}$ cells). Like innate lymphoid cells (ILC), NK

This article is published in the Special Issue CD1, MR1, NKT, and MAIT: Evolution and Origins of Non-peptidic Antigen Recognition by $T$ lymphocytes with Guest Editor Dr. Dirk Zajonc.

Dirk M. Zajonc

dzajonc@1ji.org

1 Division of Cell Biology, La Jolla Institute for Allergy and Immunology (LJ), La Jolla, CA 92037, USA

2 Department of Internal Medicine, Faculty of Medicine and Health Sciences, Ghent University, 9000 Ghent, Belgium

3 Department of Microbiology and Immunology, University of Maryland at Baltimore, Baltimore, MD 21201, USA cells, and certain memory T cells subsets, type I NKT cells can also be exclusively activated by cytokine stimulation without any TCR activation. This issue will touch on all of these aforementioned structural and functional characteristics of innate $\mathrm{T}$ cells, focusing on $\mathrm{CD} 1$ - and MR1-restricted T cells but also touching on non-classical MHC class I (MHC Ib)-restricted $\mathrm{T}$ cells for which the presented antigen is unknown. This special issue begins with the discovery and evolution of the antigen presenting molecules MR1 and CD1, and then shifts to the structural and biochemical characterization of the interaction between these non-classical class I antigen-presenting molecules and the semi-invariant TCR. Studies of innate T cell activation are tackled next, followed by a discussion of potential therapeutic applications of type I NKT cells, their role in infection and cancer, and the immune regulatory properties of type II NKT.

Keiichiro Hashimoto will recount how he and his colleagues discovered MR1 (Hashimoto 2016): the discovery of its ligands was 20 years in the making! Since its discovery by Calabi and Milstein, the CD1 molecule has been known to be ancient, thought by some to predate or at least be as old as classical class I and class II MHC molecules; Kaufman and Rogers will discuss three different models of how this most ancient nonclassical class Ib molecule might have emerged in the vertebrates, and will also describe the evolution of the natural killer (NK) receptors NKPR-1 and their ligands (Kaufman and Rogers 2016). Reinick and Van Rhijn will then illustrate the expression and gene diversification of CD1 throughout the animal kingdom (Reinick and Van Rhijn 2016).

Robert and colleagues have shown that innate-like T lymphocytes, and their recognition of class $\mathrm{Ib}$ molecules, are not simply features of warm-blooded vertebrates but also are prominent in frogs (Robert et al. 2016); these authors speculate that the innate $\mathrm{T}$ cell class $\mathrm{Ib}$ paradigm is present in all gnathostomes. MAIT cell activation, their MR1-presented 
antigens, and evolutionary aspects of the MR1-MAIT cell axis will be discussed by Lantz and colleagues (Mondot et al. 2016), while Krovi and Gapin will discuss structural and functional aspects of MAIT cell activation by MR1-presented antigens (Krovi and Gapin 2016). The structural diversification of the CD1 antigen-binding pockets and the TCR recognition of the presented glycolipid antigens will be described by Zajonc (Zajonc 2016), while Huang and Moody will discuss donor-unrestricted T cells in the human CD1 systems (Huang and Moody 2016). Many synthetic glycolipids have been designed to modulate the immune response of type I NKT cells, and Porcelli and colleagues will discuss the potential use of these novel agonists as vaccine adjuvants (Kharkwal et al. 2016). Since oxidative stress in the blood can perturb the lipidome, the question of whether human type I NKT cells can respond to these self lipid changes will be examined by Felly and Gumperz (Felley and Gumperz 2016). Since $\alpha$-GalCer, the prototypical antigen for type I NKT cells, was identified in a screen for compounds that led to the recognition and elimination of B16 melanoma cells in mice, immunotherapeutic strategies of deploying NKT cells in cancer will be discussed by Webb and colleagues (Shissler et al. 2016).

Many microbes contain non-peptidic antigens recognized by type I NKT cells, and mice lacking NKT cells show defects in the elimination of pathogens. The roles of NKT cells in the clearance of microbial infections will be discussed by Crosby and Kronenberg (Crosby and Kronenberg 2016). The different mechanisms of type I NKT cell activation, either cytokinemediated, TCR-mediated, or via an integration of both pathways, will be discussed by Brennan and colleagues, especially with regard to microbial infection (Kohlgruber et al. 2016). Since cross-talk exists between type I and type II NKT cells, Dasgupta and Kumar will discuss the immune regulatory properties of sulfatide-restricted type II NKT cells, which have recently been shown to respond to other antigens, such as certain lysophospholipids (Dasgupta and Kumar 2016). Finally, Anderson and Brossay describe how other nonclassical MHC Ib-restricted $\mathrm{T}$ cell subsets with innate-like properties exist for which the antigens are not always known, and their role during infection will be discussed by Anderson and Brossay (2016).

This special issue highlights the excellent contributions from the most active labs studying different aspects of NKT and MAIT cell biology. We would like to thank all the authors for their time and dedication in assembling this series.

Dirk M. Zajonc, Guest Editor. Martin F. Flajnik, Co-Editor. 